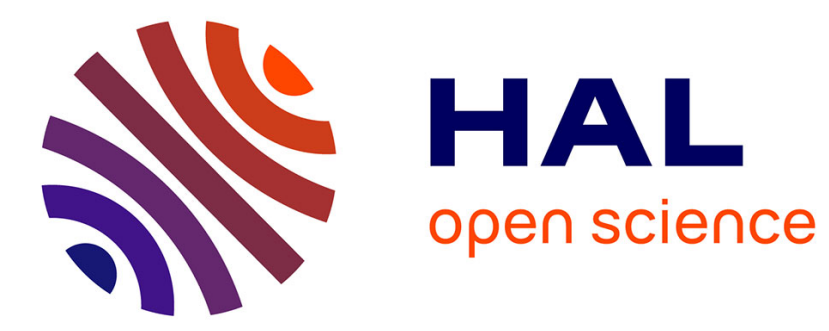

\title{
Code Replicability in Computer Graphics
}

Nicolas Bonneel, David Coeurjolly, Julie Digne, Nicolas Mellado

\section{To cite this version:}

Nicolas Bonneel, David Coeurjolly, Julie Digne, Nicolas Mellado. Code Replicability in Computer

Graphics. ACM Transactions on Graphics, 2020, 39 (4), 10.1145/3386569.3392413 . hal-02563203

\section{HAL Id: hal-02563203 \\ https://hal.science/hal-02563203}

Submitted on 5 May 2020

HAL is a multi-disciplinary open access archive for the deposit and dissemination of scientific research documents, whether they are published or not. The documents may come from teaching and research institutions in France or abroad, or from public or private research centers.
L'archive ouverte pluridisciplinaire HAL, est destinée au dépôt et à la diffusion de documents scientifiques de niveau recherche, publiés ou non, émanant des établissements d'enseignement et de recherche français ou étrangers, des laboratoires publics ou privés. 


\title{
Code Replicability in Computer Graphics
}

\author{
NICOLAS BONNEEL, Univ Lyon, CNRS, France \\ DAVID COEURJOLLY, Univ Lyon, CNRS, France \\ JULIE DIGNE, Univ Lyon, CNRS, France \\ NICOLAS MELLADO, Univ Toulouse, CNRS, France
}

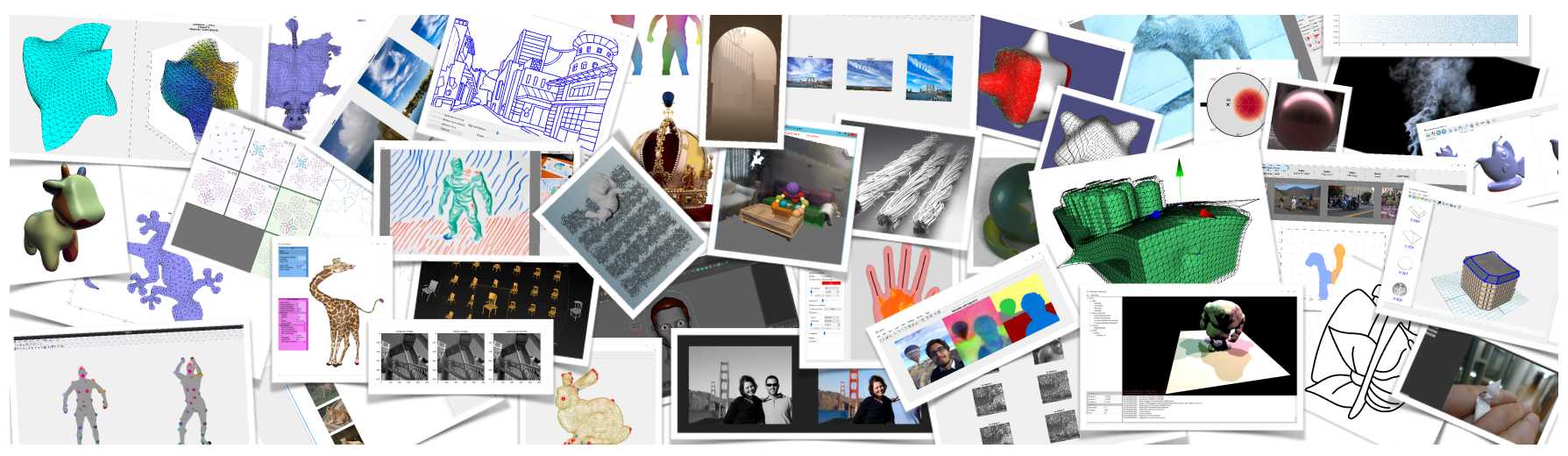

Fig. 1. We ran 151 codes provided by papers published at SIGGRAPH 2014, 2016 and 2018. We analyzed whether these codes could still be run as of 2020 to provide a replicability score, and performed statistical analysis on code sharing.

Being able to duplicate published research results is an important process of conducting research whether to build upon these findings or to compare with them. This process is called "replicability" when using the original authors' artifacts (e.g., code), or "reproducibility" otherwise (e.g., re-implementing algorithms). Reproducibility and replicability of research results have gained a lot of interest recently with assessment studies being led in various fields, and they are often seen as a trigger for better result diffusion and transparency. In this work, we assess replicability in Computer Graphics, by evaluating whether the code is available and whether it works properly. As a proxy for this field we compiled, ran and analyzed 151 codes out of 374 papers from 2014, 2016 and 2018 SIGGRAPH conferences. This analysis shows a clear increase in the number of papers with available and operational research codes with a dependency on the subfields, and indicates a correlation between code replicability and citation count. We further provide an interactive tool to explore our results and evaluation data.

Authors' addresses: Nicolas Bonneel, nicolas.bonneel@liris.cnrs.fr, Univ Lyon, CNRS, Lyon, France; David Coeurjolly, david.coeurjolly@liris. cnrs.fr, Univ Lyon, CNRS, Lyon, France; Julie Digne, julie.digne@ liris.cnrs.fr, Univ Lyon, CNRS, Lyon, France; Nicolas Mellado, nicolas. mellado@irit.fr, Univ Toulouse, CNRS, Toulouse, France.

Permission to make digital or hard copies of all or part of this work for personal or classroom use is granted without fee provided that copies are not made or distributed for profit or commercial advantage and that copies bear this notice and the full citation on the first page. Copyrights for components of this work owned by others than ACM must be honored. Abstracting with credit is permitted. To copy otherwise, or republish, to post on servers or to redistribute to lists, requires prior specific permission and/or a fee. Request permissions from permissions@acm.org.

(C) 2020 Association for Computing Machinery.

0730-0301/2020/7-ART1 $\$ 15.00$

https://doi.org/10.1145/3386569.3392413
CCS Concepts: • Computing methodologies $\rightarrow$ Computer graphics; • Software and its engineering $\rightarrow$ Open source model.

Additional Key Words and Phrases: Replicability, reproducibility, open source, code review, siggraph

\section{INTRODUCTION}

The ability to reproduce an experiment and validate its results is a cornerstone of scientific research, a key to our understanding of the world. Scientific advances often provide useful tools, and build upon a vast body of previous work published in the literature. As such, research that cannot be reproduced by peers despite best efforts often has limited value, and thus impact, as it does not benefit to others, cannot be used as a basis for further research, and casts doubt on published results. Reproducibility is also important for comparison purposes since new methods are often seen in the light of results obtained by published competing approaches. Recently serious concerns have emerged in various scientific communities from psychological sciences [Open Science Collaboration et al. 2015 ] to artificial intelligence [Hutson 2018] over the lack of reproducibility, and one could wonder about the state of computer graphics research in this matter.

In the recent trend of open science and reproducible research, this paper aims at assessing the state of replicability of papers published at ACM Transactions on Graphics as part of SIGGRAPH conferences. Contrary to reproducibility which assesses how results can be obtained by independently reimplementing published papers - an overwhelming task 
given the hundred papers accepted yearly to this event - replicability ensures the authors' own codes run and produce the published results. While sharing code is not the only available option to guarantee that published results can be duplicated by a practitioner - after all, many contributions can be reimplemented from published equations or algorithm descriptions with more or less effort - it remains an important tool that reduces the time spent in reimplementation, in particular as computer graphics algorithms get more sophisticated.

Our contributions are twofold. First, we analyze code sharing practices and replicability in computer graphics. We hypothesize strong influence of topics, an increase of replicability over time similar to the trend observed in artificial intelligence [Hutson 2018], and an increased impact of replicable papers, as observed in image processing [Vandewalle 2019]. To evaluate these hypotheses, we manually collected source codes of SIGGRAPH 2014, 2016 and 2018 papers and ran them, and when possible, assessed how they could replicate results shown in the paper or produce reasonably similar results on different inputs. Second, we provide detailed stepby-step instructions to make these software packages run (in practice, in many cases, code adaptations had to be done due to dependencies having evolved) through a website, thus becoming a large code review covering 151 codes obtained from 374 SIGGRAPH papers. We hope this platform can be used collaboratively in the future to help researchers having difficulties reproducing published results.

Our study shows that:

- Code sharing is correlated with paper citation count, and has improved over time.

- Code sharing practices largely vary with sub-fields of computer graphics.

- It is often not enough to share code for a paper to be replicable. Build instructions with precise dependencies version numbers as well as example command lines and data are important.

\section{PRIOR WORK}

The impact of research involves a number of parameters that are independent of the quality of the research itself, but of practices surrounding it. Has the peer review process been fairly conducted? Are the findings replicable? Is the paper accessible to the citizen? A number of these questions have been studied in the past within various scientific communities, which this section reviews.

Definitions. Reproducible research has been initiated in computer science [Claerbout and Karrenbach 1992] via the automation of figures production within scientific articles. Definitions have evolved [Plesser 2018] and have been debated [Goodman et al. 2016]. As per ACM standards [ACM 2016], repeatability indicates the original authors can duplicate their own work, replicability involves other researchers duplicating results using the original artifacts (e.g., code) and hardware, and reproducibility corresponds to other researchers duplicating results with their own artifacts and hardware - we will hence use this definition. We however mention that various actors of replicable research have advocated for the opposite definition: replicability being about answering the same research question with new materials while reproducibility involves the original artifacts [?] - a definition championed by the National Academies of Sciences [?].

Reproducibility and replicability in experimental sciences. Concerns over lack of reproducibility have started to emerge in several fields of studies, which has led to the term "reproducibility crisis" [Pashler and Harris 2012]. In experimental sciences, replicability studies evaluate whether claimed hypotheses are validated from observations (e.g., whether the null hypothesis is consistently rejected and whether effect sizes are similar). In different fields of psychology and social sciences, estimations of replication rates have varied between $36 \%$ out of 97 studies with significant results, with half the original effect size [Open Science Collaboration et al. 2015], $50 \%-54 \%$ out of 28 studies [Klein et al. 2018], $62 \%$ out of 21 Nature and Science studies with half the original effect size [Camerer et al. 2018], and up to roughly $79 \%$ out of 342 studies [Makel et al. 2012]. In oncology, a reproducibility rate of $11 \%$ out of 53 oncology papers has been estimated [Begley and Ellis 2012], and a collaboration between Science Exchange and the Center for Open Science (initially) planned to replicate 50 cancer biology studies [Baker and Dolgin 2017]. Over 156 medical studies reported in newspapers, about $49 \%$ were confirmed by meta-analyses [Dumas-Mallet et al. 2017]. A survey published in Nature [Baker 2016] showed large disparities among scientific fields: respondents working in engineering believed an average of $55 \%$ of published results are reproducible $(N=66)$, while in physics an average of $73 \%$ of published results were deemed reproducible $(N=91)$.

This has resulted in various debates and solutions such as reducing hypothesis testing acceptance thresholds to $p<$ 0.005 [Benjamin et al. 2018] or simply abandoning hypothesis testing and p-values as binary indicators [McShane et al. 2019], providing confidence intervals and using visualization techniques [Cohen 2016], or improving experimental protocols [Begley 2013].

While computer graphics papers occasionally include experiments such as perceptual user studies, our paper focuses on code replicability.

Reproducibility and replicability in computational sciences. In hydrology, Stagge et al. [2019] estimate via a survey tool that $0.6 \%$ to $6.8 \%$ of 1,989 articles (95\% Confidence Interval) can be reproduced using the available data, software and code - a major reported issue being the lack of directions to use the available artifacts (for $89 \%$ of tested articles). High energy physicists, who depend on costly, often unique, experimental setups (e.g., the Large Hadron Collider) and produce enormous datasets, face reproducibility challenges both in data collection and processing [Chen et al. 2019]. Such challenges are tackled by rigorous internal review processes before data and tools are opened to larger audiences. It is argued that analyses should be automated from inception and 
not as an afterthought. Closer to our community is the replication crisis reported in artificial intelligence [Gundersen and Kjensmo 2018; Hutson 2018]. Notably, the authors surveyed 400 papers from top AI conferences IJCAI and AAAI, and found that $6 \%$ of presenters shared their algorithm's code, $54 \%$ shared pseudo-code, $56 \%$ shared their training data, and $30 \%$ shared their test data, while the trend was improving over time. In a recent study on the reproducibility of IEEE Transactions on Image Processing papers [Vandewalle 2019], the authors showed that, on average, code availability approximately doubled the number of citations of published papers. Contrary to these approaches, we not only check for code availability, but also evaluate whether the code compiles and produces similar results as those found in the paper, with reasonable efforts to adapt and debug codes when needed.

Efforts to improve reproducibility are nevertheless flourishing from early recommendations such as building papers using Makefiles in charge of reproducing figures [Schwab et al. 2000] to various reproducibility badges proposed by ACM [ACM 2016] in collaboration with the Graphics Replicability Stamp Initiative [Panozzo 2016]. Colom et al. list a number of platforms and tools that help in reproducible research [2018]. Close to the interest of the computer graphics community, they bring forward the IPOL journal [Colom et al. 2015] whose aim is to publish image processing codes via a web interface that allows to visualize results, along with a complete and detailed peer-reviewed description of the algorithm. They further mention an initiative by GitHub [2016] to replicate published research, though it has seen very limited success (three replications were initiated over the past three years). In Pattern Recognition, reproducible research is awarded with the Reproducible Label in Pattern Recognition organized by the biennal Workshop on Reproducible Research in Pattern Recognition [Kerautret et al. 2019, 2017]. Programming languages and software engineering communities have created the Artifact Evaluation Committees for accepted papers [?], with incentives such as rewarding with additional presentation time at the conference and an extra page in the proceedings, with special recognition for best efforts.

Other initiatives include reproducibility challenges such as the one organized yearly since 2018 by the ICLR conference in machine learning [Pineau et al. 2019] that accepts submissions aiming at reproducing published research at ICLR. In 2018, reproducibility reports of 26 ICLR papers were submitted, out of which 4 were published in the ReScience $\mathrm{C}$ journal.

Open access. Software bugs have had important repercussions on collected data and analyses, hence pushing for open sourcing data and code. Popular examples include Microsoft Excel that converts gene names such as SEPT2 (for Septin 2) to dates [Ziemann et al. 2016], or a bug in widely used fMRI software packages that resulted in largely inflated falsepositive rates, possibly affecting many published results [Eklund et al. 2016]. Recently, Nature Research has enforced an open data policy [nat 2018], stated in their policies as authors are required to make materials, data, code, and associated protocols promptly available to readers without undue qualifications, and proposes a journal focused on sharing high re-use value data called Scientific Data [Scientific Data (Nature Research) 2014]. Other platforms for sharing scientific data include the Open Science Framework [Center for Open Science 2015]. Related to code, Colom et al. [2018] reports the websites mloss that lists machine learning codes, RunMyCode for scientists to share code associated with their research paper, or ResearchCompendia that stores data and codes. Long-term code availability is also an issue, since authors' webpages are likely to move according to institution affiliations so that code might be simply unavailable. Code shared on platforms such as GitHub is only available as long as the company exists which can also be an issue, if limited. For long-term code storage, the Software Heritage initiative [Di Cosmo and Zacchiroli 2017] aims at crawling the web and platforms such as GitHub, Bitbucket, Google code etc. for open source software and stores them in a durable way. Recently, the Github Archive Program [Github 2020] pushed these ideas further and propose a pace layer strategy where code is archived at different frequencies (real-time, monthly, every 5 years), with advertised lifespans up to 500 years and possibly 10,000 years.

Other assessments of research practices. Reproducibility of paper acceptance outcome has been assessed in machine learning. In 2014, the prestigious NIPS conference (now NeurIPS) has performed the NIPS consistency experiment: a subset of 170 out of 1678 submissions were assigned to two independent sets of reviewers, and consistency between reviews and outcomes were evaluated. The entire process, results, and analyses, were shared on an open platform [nip 2014]. Decisions were inconsistent for 43 out of 166 reviewed papers ( 4 were withdrawn, 101 were rejected by both committees, 22 were accepted by both committees). Other initiatives for more transparent processes include the sharing of peer reviews of published papers on platforms such as OpenReview [Soergel et al. 2013] or directly by journals [The Royal Society Publishing 2020], and the post-publication monitoring for misconducts or retractions on platforms such as PubPeer and RetractionWatch [Didier and Guaspare-Cartron 2018].

\section{METHOD}

Our goal is to assess trends in replicability in computer graphics. We chose to focus on the conference in the field with highest exposure, ACM SIGGRAPH, as an upper bound proxy for replicability. Although this hypothesis remains to be verified, this conference more often publishes completed research projects as opposed to preliminary exploratory ideas that are more often seen in smaller venues which could explain lower code dissemination. To estimate a trend over time, we focus on three SIGGRAPH conferences: SIGGRAPH 2014 (Vancouver, 127 accepted papers), 2016 (Anaheim, 119 accepted papers), and 2018 (Vancouver, 128 accepted papers). We did not include SIGGRAPH 2019 (Los Angeles) since authors sometimes need time to clean up and publish their code after publication. We did not include SIGGRAPH Asia nor 
papers published in ACM Transactions on Graphics outside of the conference main track to reduce variability in results and keep a more focused scope. We chose a two-year interval between conferences in the hope to get clearer trends, and to keep a tractable number of papers to evaluate.

We searched for source codes as well as closed-source binaries for all papers. We restricted our search to original implementations and reimplementations authored and released by the original authors of the paper, excluding reimplementations by others, as we aim at assessing replicability and not reproducibility (see Sec. 2). For each paper, we report the objective and subjective information described below.

Identifying and factual information. This includes the paper name and DOI, ACM keywords, pdf, project and code or binaries URLs if they have been found, as well as information indicating if authors are from the industry, academia, or unaffiliated, for further analysis. For papers, we include information as whether they can be found on arXiv or other Open Archive Initiative providers we may have found, in open access on the ACM Digital Library, or by other means such as institutional web pages. Aside from ACM keywords, we further categorize papers into 6 broad topics related to computer graphics, and we also keep track of whether they relate to neural networks. We defined these topics as:

- Rendering. This includes simulating light transport, real-time rendering, sampling, reflectance capture, datastructures for intersections, and non-photorealistic rendering.

- Animation and simulation. This includes character animation, motion capture and rigging/skinning, cinematography/camera path planning, deformable models as well as fluid, cloth, hair or sound simulation, including geometric or topology problems related to these subjects.

- Geometry. This includes geometry processing and modeling, for point-based, voxel-based and mesh-based geometries, as well as topology, mapping, vector fields and shape collection analysis. We also include image-based modeling.

- Images. This includes image and video processing, as well as texture synthesis and editing, image segmentation, drawing, sketching and illustration, intrinsic decomposition or computational photography. We also included here image-based rendering, which relies more on image techniques than rendering.

- Virtual Reality. This category includes virtual and augmented reality, 3d displays, and interactions.

- Fabrication. This includes 3d printing, knitting or caustic design.

We strive to classify each paper into a single category to simplify analyses. Both these categories and paper assignments to these categories can be largely debated. While they may be prone to errors at the individual level, they still provide meaningful insight when seen as statistical aggregates. These categories were used in our analysis instead of ACM keywords

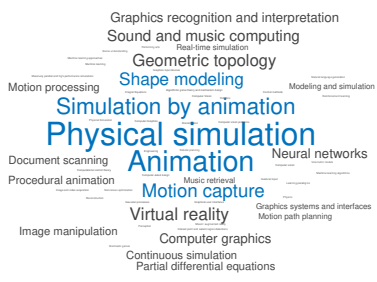

(a) Animation

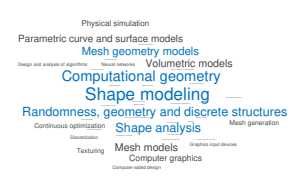

(c) Geometry

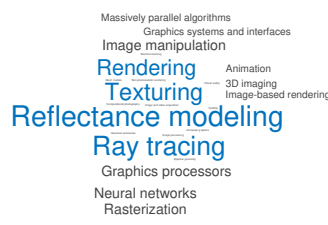

(e) Rendering

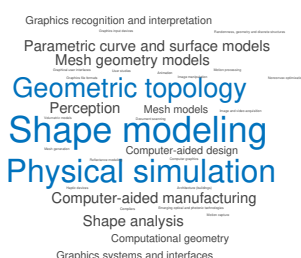

(b) Fabrication

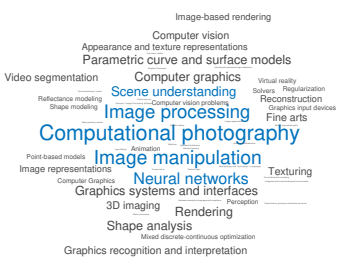

(d) Image

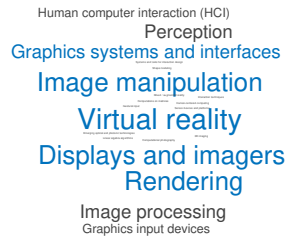

(f) Virtual Reality
Fig. 2. Distribution of the ACM keywords per topic. The font size reflects the number of papers associated with a keyword.

for several reasons: first, we counted more than 127 different ACM keywords which would make overspecialized categories. The hierarchical nature of this taxonomy also makes the analysis more complicated. In Fig. 2 we show the distribution of ACM keywords of papers involved in each of our categories. Interestingly, this visualization exacerbates the lack of ACM keywords dedicated to fabrication despite the increasing popularity of this topic.

Information about code includes code license, presence of documentation, readme files and explicit mention of the code authors (who usually are a subset of the paper authors), as well as build mechanism (Makefile, CMakeLists, SCons, IDE projects, or other types of scripts), and lists of dependencies. We notably indicate whether library or software dependencies are open source (e.g., Eigen, OpenCV), closed source but free at least for research purpose (e.g., mosek, CUDA or Intel MKL), or closed source and paying even for research purpose (e.g., Matlab). Similarly, we ask whether the code depends on data other than examples or input data (e.g., training data or neural network description files) and their license.

One of our key contributions is that we report the undocumented steps required to make the code run - from bug fixes to dependency installation procedures. We believe this information is valuable to the community as these steps are 
often independently found by students relying on these codes sometimes after significant effort.

Subjective judgments on replicability. For papers without published code, this includes information as to whether the paper contains explicit algorithms and how much effort is deemed required to implement them (on a scale of 1 to 5 ). For algorithms requiring little reimplementation effort (with a score of 5) - typically for short shaders or short self-contained algorithms - this can give an indication as to why releasing the code was judged unnecessary. For papers containing code, we evaluate how difficult it was to replicate results through a number of questions on a scale of 1 to 5 . This includes the difficulty to find and install dependencies, to configure and build the project, to fix bugs, to adapt the code to other contexts, and how much we could replicate the results shown in the paper. We strived to remain flexible in the replicability score: often, the exact input data were not provided but the algorithms produced satisfactory results that are qualitatively close to those published on different data, or algorithms relied on random generators (e.g., for neural network initializations) that do not produce repeatable number sequences and results. Contrary to existing replicability initiatives, we did not penalize these issues, and this did not prevent high replicability scores.

We shared the task of evaluating these 374 submissions across 4 full-time tenured researchers (authors of the paper), largely experienced in programming and running complex computer graphics systems. Reasonable efforts were made to find and compile the provided code, including retrieving outdated links from the WayBack Machine [Tofel 2007], recreating missing Makefiles, debugging, trying on multiple OS (compiling was tested on Windows 10, Debian Buster, Ubuntu 18.04 and 19.10 and MacOS $10.15^{1}$ ), or adapting the code to match libraries having evolved. Efforts to adapt the code to evolved libraries, compilers or languages are due to practical reasons: it is sometimes impractical to rely on old Visual Studio 2010 precompiled libraries when only having access to a newer version, or to rely on TensorFlow 1.4 .0 requiring downgrading CUDA drivers to version 8 for the sole purpose of having a single code run. We chose to avoid contacting authors for clarifications, instructions or to report bug fixes to protect anonymity. We also added the GitHub projects to Software Heritage [Di Cosmo and Zacchiroli 2017] when they were not already archived and gave the link to the Software Heritage entry in our online tool.

\section{DATA EXPLORATION}

We provide the data collected during our review as a JSON file, available as supplementary material. Each JSON entry describes the properties of a paper (e.g., author list, project page, ACM keywords, topics) and its replicability results (e.g., scores, replicability instructions). All the indicators and statistics given in this paper are computed from this data,

\footnotetext{
${ }^{1}$ Ubuntu 14.04 and Windows 2012 virtual machines for very specific tests.
}

and we provide in supplementary materials all the scripts required to replicate our analysis.

We facilitate data exploration by providing an intuitive web interface available at https://replicability.graphics (see Fig. 3) to visualize collected data. This interface allows two types of exploration, either the whole dataset or per paper.

Dataset exploration. Our dataset exploration tool is split into two components: a table listing the reviewed papers, and two graphs showing statistics about the table content. A first graph displays the distribution of papers with respect to the code/pseudocode availability, and their replicability score. A second graph shows papers availability, either as ACM Open Access or as a preprint provided by the authors. The interactive table allows to filter the dataset by the author name, paper title, publication year and/or by topic, and to update the graphs according to the selection. It is also possible to sort the paper by their properties and in particular their replicability score or a documentation score between 0 and 2 ( 0 : no documentation, 2: exhaustive documentation). Each paper is associated with a dedicated webpage accessible directly from the table.

Per-paper exploration. The paper webpage gives a direct access to the information extracted from the JSON file. It includes the links to resources available online (Digital ACM library, preprint, code), several information (e.g., paper topic, nature of the artifact, list of the dependencies) and a breakdown of the replicability experiment when code was available (scores and comments). In addition, the paper webpage gives the Altmetric Attention Score ${ }^{2}$ and links to the Altmetric webpage of the paper if available. This score measures the overall attention a paper has received, including on social networks, which differs from academic citation scores. The comment section mostly covers the steps that the reviewer had to follow in order to try to replicate the paper, which includes details about the dependencies management and updates, bug fixes or code modifications. We expose the exact revision number (for git projects) or MD5 hash of the archive file (for direct download) of codes that relate to the comments. The website allows for commenting scores and instructions, both as a user and as a paper author, as well as adding new entries, for adding new or updated codes.

\section{RESULTS AND ANALYSIS}

This section analyzes both objective and subjective metrics. All reported p-values were adjusted for multiple comparisons using the false discovery rate control procedure proposed by Benjamini and Hochberg [1995].

\subsection{Objective analysis}

Availability of papers. Papers are overall available. Over all 374 papers, only two are available only on the ACM Digital Library. Notably, ACM provides free access to all SIGGRAPH and SIGGRAPH Asia proceedings though it

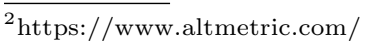



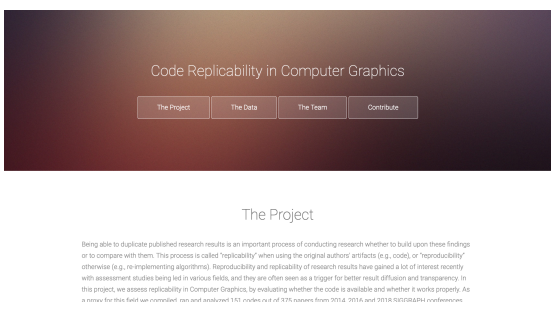
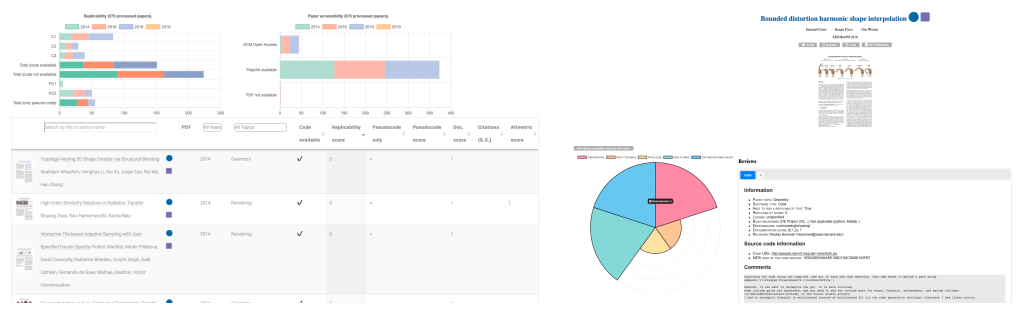

Fig. 3. We designed a web interface to explore our collected data allowing to see individual paper replicability and build instructions, available at https://replicability.graphics.

is little advertised [?]. Also, 27 are available as preprints on $\operatorname{arXiv}$ (9 only on arXiv), 17 on HAL ( 7 only on HAL $)^{3}$ 44 benefit from the ACM Open Access policy -the other papers being available as preprints at least on the authors website or other paper repositories.

Availability of code. Software packages were available for 151 papers, which consist of 133 papers for which source code was provided plus 18 papers for which no source code was provided but instead compiled software was provided. For the rest of the analysis, we considered both compiled and open source software combined. While open source research codes allow for adaptation, making it easier to build upon them, and are thus ideal, binary software at least allows for effortless method comparisons. Nevertheless, among these software packages, we could not run 19 of them due to technical issues preventing the codes to compile or run, and 5 of them due to lack of dedicated hardware (see Sec. 6). Among these 133 codes, 60 do not have license information, which could notably prevent code dissemination in the industry, and 11 do not come with any documentation nor build instructions.

We perform $\chi^{2}$ tests to analyze trends in code sharing. Overall, codes or binaries could be found for 37 papers out of $127(29.1 \%)$ in 2014,47 out of $119(39.5 \%)$ in 2016, 67 papers out of $128(52.3 \%)$ in 2018. This increase is statistically significant between 2014 and $2018\left(p=1.310^{-3}\right)$, though not between 2014 and $2016(p=0.13)$ nor between 2016 and $2018(p=0.086)$. This trend is similar to that observed in artificial intelligence [Gundersen and Kjensmo 2018; Hutson 2018]. Results can be seen in Fig. 4. In two cases, we had to retrieve the code from the WayBack Machine [Tofel 2007] due to expired URLs. Further analysis per topic shows vastly different practices, with $17.1 \%$ of papers sharing code for Fabrication, 26.9\% for Animation, 31.8\% for Virtual Reality, $47.9 \%$ for Rendering, $51.9 \%$ for Geometry and $57.9 \%$ for Images (Fig. 4).

We also analyzed the involvement of at least one author from the industry on the release of codes or binaries. We found that overall, papers involving the industry provided code or binaries $31.3 \%$ of the times, while this was the case for $45.4 \%$ of purely academic papers - a difference that is significant $(p=0.031)$. This could be explained by strict

\footnotetext{
${ }^{3}$ Some references or preprints may also be available on other OAI providers thanks to database interconnections or local initiatives, we only report here the most significant ones found by this study.
}
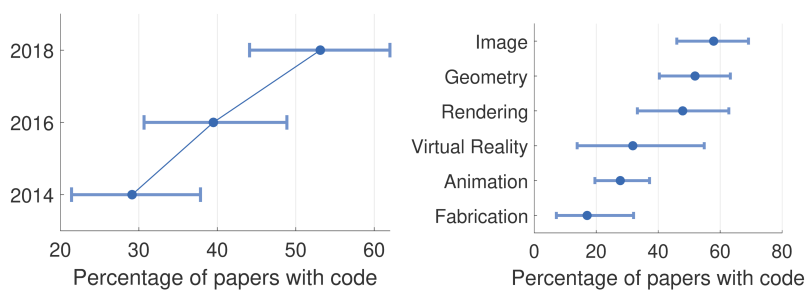

Fig. 4. We compute the percentage of papers that include either code or binaries as a function of years and topic. We also show ClopperPearson $95 \%$ confidence intervals.

rules imposed by employers, understandably worried about industrial consequences of sharing a code.

Given the sheer amount of deep learning codes available online, we hypothesized that deep learning-related papers were more likely to share code. We tested this hypothesis on our dataset, but we found that they provided code only $44.6 \%$ of the times (25 out of 56 ), while this was the case $39.6 \%$ of the times for non-deep papers (126 out of 318 ) - a non-significant difference $(p=0.48)$.

We finally found that, in the long term, sharing code results in higher citations, with a median citation count of up to 67 in 2014 for papers sharing code compared to 43 for papers not sharing code (see Fig. 5). A Mann-Whitney U-test gives this difference significant $(p=0.045)$. This observation is similar to that observed in image processing [Vandewalle 2019] though the effect is less pronounced (they observed a doubling of citation rates). Few additional information is given in Table 1.

\subsection{Subjective analysis}

As replicability scores are subjective, we first perform an analysis of variance (ANOVA), despite some limitations here (see Norman [2010] for a full discussion), aimed at determining two things: is there a dependence on the reviewer of the code on replicability scores? And, does the year influence replicability (as it would seem that older non-maintained codes are harder to replicate)? The ANOVA is performed on the replicability score, taking only papers with codes for which compiling was successful, and with two factors: reviewer and year. The answer to both questions seems negative (resp. $p=0.13$ and $p=0.27)$.

To make the codes run, we had to modify source codes in 68 out of 151 codes. These code alterations were deemed 


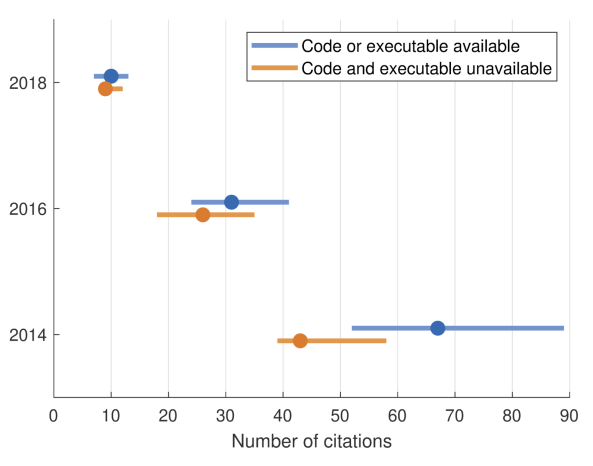

Fig. 5. We compute the median number of citations and its $95 \%$ confidence intervals for papers sharing code (or executable) and for papers not sharing code nor executable.

Table 1. Additional quantitative data from our study.

\begin{tabular}{l|c|c|c|r} 
& 2014 & 2016 & 2018 & Total \\
\hline Nb of papers & 127 & 119 & 128 & 374 \\
Nb of papers with codes & 37 & 47 & 67 & 151 \\
\hline \hline Nb of codes without license & 16 & 24 & 20 & 60 \\
Nb of codes with doc. score 0 & 6 & 16 & 14 & 36 \\
Nb of codes flagged as "Deep" & 4 & 4 & 17 & 25 \\
Nb of codes with associated data & 5 & 7 & 18 & 30 \\
\hline
\end{tabular}

difficult ("easy to fix bugs" score $\leq 2$ out of 5 ) for 20 codes. The time spent to make codes run, including time to debug and compile dependencies was longer than 100 minutes for 27 codes.

In the years covered by this study, we found a total of 5 papers with a Replicability Stamp from the Graphics Replicability Stamp Initiative [Panozzo 2016]. While this number is too low to derive meaningful statistics, one can note that out of these 5 papers, 4 get the maximum score for results replication. This could be expected because this initiative ensures that a script for each single result shown in the paper is provided. A limitation is that these scripts are only guaranteed to work at the time when the stamp is granted -a limitation shared by the present study.

\section{LIMITATIONS}

Our analysis has a number of limitations. First, the data we collected may only be partly reliable. While we spent reasonable efforts to find, run and compile codes, it is possible that we missed codes, or that additional efforts or contacting the authors for clarifications or to report bugs would result in different outcome for a few papers. Similarly, we could not fully evaluate codes that depend on specific hardware (such as spatial light modulators, microcontrollers, Hall effect sensors etc.) for 4 papers. Our analysis focused on assessing the codes provided by the authors which only assesses replicability but not reproducibility: there are instances for which papers were successfully reimplemented by other teams, which falls out of our analysis scope. It could also be expected that certain codes could be available upon request ; in fact, in a few cases, the provided code relied on data only available upon request, which we did not assess.

Second, the codes we found and assessed may have evolved after the paper has been published, which we cannot control. Similarly, the published code could be a cleaned-up version of the original code, or even a full reimplementation.

Third, our focus on SIGGRAPH could hide a more negative picture of the entire field. We believe that the exposure SIGGRAPH probably gives biases our results, with a tendency to find more codes here than in smaller venues. It would be an interesting future work to compare replicability across computer graphics venues.

\section{RECOMMENDATIONS}

Our replicability evaluation led us to identify a number of issues. First, the number of dependencies was often correlated with the difficulty to compile - especially on Windows. Precompiled libraries were sometimes provided for compilers that became outdated, or some dependencies were no longer supported on recent hardware or OS. The lack of precise dependencies version number was another important issue we faced. Package managers for Python such as pip or conda evolve and default to different library versions, and build instructions or installation scripts did not directly work with these new versions. Lack of instructions for running software raised important frustration: default parameters were sometimes not provided, command line parameters not described, or results output as numerical values in the console or written to files of undocumented format with no clear ways to use. In one case we had to develop a software for reading the output file and displaying results. Similarly, input data were not always provided, or sometimes only provided upon request. Finally, in some cases, the software implemented only part of the method, producing results that did not match the quality of the results shown in the paper (e.g., missing post-processing, or only implementing the most important parts of the paper).

This leads us to issue several recommendations for the research community in Computer Graphics to promote their research work through the code replicability point of view.

For the authors. Sharing the code or any artifact to help the replicability of the paper results is a good way to spread the contributions of the paper, as shown in terms of citation numbers in Sec. 5.1 and independently by Vandewalle [2019]. When shipping the code as a supplementary material for the paper, several concerns must be addressed: Accessibility of the source code (e.g., using the Software Heritage archive when the article is accepted) ; Replicability of the build process specifying the exact versions of the software or libraries that must be used to build and execute the code (for instance 
using container/virtualization services - docker ${ }^{4}$ - or package/configurations managements services -anaconda ${ }^{5}, \mathrm{Nix}^{6}$ etc.); Clarity of the source code as a knowledge source (e.g., through technical documentation and comments in the code); and, finally, tractability of the coding process (authorship, clear licensing etc.). Extra care should be given to codes that depend on rapidly evolving libraries. This is particularly the case of deep learning libraries (TensorFlow, Pytorch, Caffe etc.). As an example, several syntax changes occurred in pytorch over the past few years and caffe appears not to be maintained anymore (e.g., pre-built binaries are provided up to Visual Studio 2015, and the last commit on Github was in March 2019) ; Python 2.7 is not maintained anymore as of January 1st, 2020. We recommend limiting the number of dependencies when possible - e.g., avoiding to depend on large libraries for the sole purpose of loading image files possibly shipping dependencies with the source code (with integration into the project build framework). Similarly, deep learning codes can require up to several days of training: sharing pre-trained models together with the training routines is a good way to ensure replicability.

For the conference program chairs. Not all research papers need to be involved in a source code replicability effort. A paper presenting a mathematical proof of the asymptotic variance of some sampler is intrinsically reproducible and does not need source code. On the contrary, for research papers for which it would make sense, the source code should be considered as a valuable artifact when evaluating a submission. This can be emphasized and encouraged in the guidelines and submission forms. Asking the reviewers to check the replicability of the article through the provided code is an ultimate goal for targeting replicability but it may not be sustainable for the entire community. Intermediate action could be to communicate about the importance of paper replicability and to allow authors to submit their (anonymous) code and data with appropriate entries in the submission system. Furthermore, we advocate for a specific deadline for the submission of the code and data materials, e.g. one week after the paper deadline. The objective would be to let additional time for authors to sanitize their code and encourage its publication, without interfering with the intense paper polishing process happening right before the paper deadline, nor with the reviewing process, since reviewers would only wait for a short amount of time before getting the code.

For the publishers. Publishers already offer the possibility to attach supplementary materials to published papers (videos, source code, data...). Beside videos, other types of supplementary documents are not clearly identifiable. We would recommend to clearly tag (and promote on the publisher library) supplementary data that correspond to source codes. Independent platforms, such as Software Heritage [Di Cosmo

\footnotetext{
${ }^{4}$ https://www.docker.com

${ }^{5}$ https://anaconda.org

${ }^{6}$ https://nixos.org
}

and Zacchiroli 2017], permit archiving and attach unique identifiers to source codes as well as a timestamp. Publishers could easily rely on these platforms to link to the source code of a paper.

\section{CONCLUSION}

Our in-depth study of three years of ACM SIGGRAPH conference papers showed a clear increase in replicability as authors are increasingly sharing their codes and data. Our study also showed that sharing code was correlated with a higher impact, measured in terms of citation numbers. We developed a website which aims at helping practitioners run existing codes on current hardware and software generations, with build instructions for 151 codes we found online when we could run them. Contrary to existing replicability stamps, our replicability scores are non-binary but on a 1-to-5 integer scale, less strict in the sense that results sufficiently close those shown in the paper on different data were deemed appropriate, but sometimes inconsistent with these stamps when software could not be run anymore on current hardware and software generations. In the future, we hope to see interactions with these replicability stamp initiatives for which we share the common goal of spreading open research.

\section{ACKNOWLEDGMENTS}

We thank Roberto Di Cosmo for insightful discussions and the reviewers for their constructive feedback. This work was funded in part by ANR-16-CE23-0009 (ROOT), ANR-16CE33-0026 (CALiTrOp), ANR-15-CE40-0006 (CoMeDiC) and ANR-16-CE38-0009 (e-ROMA).

\section{REFERENCES}

ACM. 2016. Artifact Review and Badging. https://www.acm.org/ publications/policies/artifact-review-badging (accessed Dec. 2019).

Monya Baker. 2016. 1,500 scientists lift the lid on reproducibility. Nature News 533, 7604 (2016), 452.

Monya Baker and Elie Dolgin. 2017. Cancer reproducibility project releases first results. Nature News 541, 7637 (2017), 269.

C Glenn Begley. 2013. Reproducibility: Six red flags for suspect work. Nature 497, 7450 (2013), 433.

C Glenn Begley and Lee M Ellis. 2012. Drug development: Raise standards for preclinical cancer research. Nature 483, 7391 (2012), 531.

Daniel J Benjamin, James O Berger, Magnus Johannesson, Brian A Nosek, E-J Wagenmakers, Richard Berk, Kenneth A Bollen, Björn Brembs, Lawrence Brown, Colin Camerer, et al. 2018. Redefine statistical significance. Nature Human Behaviour 2, 1 (2018), 6.

Yoav Benjamini and Yosef Hochberg. 1995. Controlling the false discovery rate: a practical and powerful approach to multiple testing. Journal of the Royal statistical society: series $B$ (Methodological) 57, 1 (1995), 289-300.

Colin F Camerer, Anna Dreber, Felix Holzmeister, Teck-Hua Ho, Jürgen Huber, Magnus Johannesson, Michael Kirchler, Gideon Nave, Brian A Nosek, Thomas Pfeiffer, et al. 2018. Evaluating the replicability of social science experiments in Nature and Science between 2010 and 2015. Nature Human Behaviour 2, 9 (2018), 637.

Center for Open Science. 2015. Open Science Framework. https: //www.osf.io (accessed Dec. 2019).

Xiaoli Chen, Sünje Dallmeier-Tiessen, Robin Dasler, Sebastian Feger, Pamfilos Fokianos, Jose Benito Gonzalez, Harri Hirvonsalo, Dinos Kousidis, Artemis Lavasa, Salvatore Mele, et al. 2019. Open is not enough. Nature Physics 15, 2 (2019), 113-119. 
Jon F Claerbout and Martin Karrenbach. 1992. Electronic documents give reproducible research a new meaning. In SEG Technical Program Expanded Abstracts 1992. Society of Exploration Geophysicists, 601-604.

Jacob Cohen. 2016. The earth is round ( $\mathrm{p}_{\mathrm{i}}$. 05). In What if there were no significance tests? Routledge, 69-82.

Miguel Colom, Bertrand Kerautret, and Adrien Krähenbühl. 2018. An Overview of Platforms for Reproducible Research and Augmented Publications. In International Workshop on Reproducible Research in Pattern Recognition. Springer, 25-39.

Miguel Colom, Bertrand Kerautret, Nicolas Limare, Pascal Monasse, and Jean-Michel Morel. 2015. IPOL: a new journal for fully reproducible research; analysis of four years development. In 2015 Ith International Conference on New Technologies, Mobility and Security (NTMS). IEEE, 1-5.

Roberto Di Cosmo and Stefano Zacchiroli. 2017. Software Heritage: Why and How to Preserve Software Source Code. In iPRES 2017 . 14 th International Conference on Digital Preservation (2017-09-25). Kyoto, Japan.

Emmanuel Didier and Catherine Guaspare-Cartron. 2018. The new watchdogs vision of science: A roundtable with Ivan Oransky (Retraction Watch) and Brandon Stell (PubPeer). Social studies of science 48, 1 (2018), 165-167.

Estelle Dumas-Mallet, Andy Smith, Thomas Boraud, and François Gonon. 2017. Poor replication validity of biomedical association studies reported by newspapers. PloS one 12, 2 (2017), e0172650.

Anders Eklund, Thomas E Nichols, and Hans Knutsson. 2016. Cluster failure: Why fMRI inferences for spatial extent have inflated falsepositive rates. Proceedings of the national academy of sciences 113 , 28 (2016), 7900-7905.

Github. 2016. Call for replication repository. https://github.com/ ReScience/call-for-replication (accessed Dec. 2019).

Github. 2020. Github Archive Program. https://archiveprogram.github. com/ (accessed Jan. 2020).

Steven N Goodman, Daniele Fanelli, and John PA Ioannidis. 2016 What does research reproducibility mean? Science translational medicine 8, 341 (2016)

Odd Erik Gundersen and Sigbjørn Kjensmo. 2018. State of the art: Reproducibility in artificial intelligence. In Thirty-Second AAAI Conference on Artificial Intelligence.

Matthew Hutson. 2018. Artificial intelligence faces reproducibility crisis.

Bertrand Kerautret, Miguel Colom, Daniel Lopresti, Pascal Monasse, and Hugues Talbot. 2019. Reproducible Research in Pattern Recognition. Springer.

Bertrand Kerautret, Miguel Colom, and Pascal Monasse. 2017. Reproducible Research in Pattern Recognition. Springer.

Richard A Klein, Michelangelo Vianello, Fred Hasselman, Byron G Adams, Reginald B Adams Jr, Sinan Alper, Mark Aveyard, Jordan R Axt, Mayowa T Babalola, Štěpán Bahník, et al. 2018. Many Labs 2 : Investigating variation in replicability across samples and settings. Advances in Methods and Practices in Psychol. Sci. 1, 4 (2018).

Neil D. Lawrence. 2014. Managing Microsoft's Conference Management Toolkit with Python for NIPS 2014. https://github.com/sods/ conference (accessed Apr. 2020).

Matthew C Makel, Jonathan A Plucker, and Boyd Hegarty. 2012 Replications in psychology research: How often do they really occur? Perspectives on Psychological Science 7, 6 (2012), 537-542.

Blakeley B McShane, David Gal, Andrew Gelman, Christian Robert, and Jennifer L Tackett. 2019. Abandon statistical significance. The American Statistician 73, sup1 (2019), 235-245.

Nature. 2018. Reproducibility: let's get it right from the start. $N a$ ture Communications 9, 1 (2018), 3716. https://doi.org/10.1038/ s41467-018-06012-8

Geoff Norman. 2010. Likert scales, levels of measurement and the laws of statistics. Advances in health sciences education 15, 5 (2010), 625-632.

Open Science Collaboration et al. 2015. Estimating the reproducibility of psychological science. Science 349, 6251 (2015), aac4716.

Daniele Panozzo. 2016. Graphics replicability stamp initiative. http //www.replicabilitystamp.org/ (accessed Dec. 2019).

Harold Pashler and Christine R Harris. 2012. Is the replicability crisis overblown? Three arguments examined. Perspectives on Psychological Science 7, 6 (2012), 531-536.

Joelle Pineau, Koustuv Sinha, Genevieve Fried, Rosemary Nan Ke, and Hugo Larochelle. 2019. ICLR Reproducibility Challenge 2019.
ReScience $C$ (2019).

Hans E Plesser. 2018. Reproducibility vs. replicability: a brief history of a confused terminology. Frontiers in neuroinformatics 11 (2018), 76 .

Matthias Schwab, N Karrenbach, and Jon Claerbout. 2000. Making scientific computations reproducible. Computing in Science \& Engineering 2, 6 (2000), 61-67.

Scientific Data (Nature Research). 2014. Recommended Data Repositories. https://www.nature.com/sdata/policies/repositories (accessed Dec. 2019).

David Soergel, Adam Saunders, and Andrew McCallum. 2013. Open Scholarship and Peer Review: a Time for Experimentation. ICML 2013 Peer Review Workshop (2013). https://openreview.net/ (accessed Dec. 2019)

James H Stagge, David E Rosenberg, Adel M Abdallah, Hadia Akbar, Nour A Attallah, and Ryan James. 2019. Assessing data availability and research reproducibility in hydrology and water resources. Scientific data 6 (2019).

The Royal Society Publishing. 2020. Open Peer Review. https: //royalsocietypublishing.org/rspa/open-peer-review (accessed Jan 2020).

Brad Tofel. 2007. Waybackfor accessing web archives. In Proceedings of the 7th International Web Archiving Workshop. 27-37.

Patrick Vandewalle. 2019. Code availability for image processing papers: a status update. https://lirias.kuleuven.be/retrieve/ 541895\$DVandewalle_onlinecode_TIP_SITB19.pdf

Mark Ziemann, Yotam Eren, and Assam El-Osta. 2016. Gene name errors are widespread in the scientific literature. Genome biology 17,1 (2016), 177 